\title{
Ras protein expression as a marker for breast cancer
}

\author{
GLORIA M. CALAF ${ }^{1,2}$ and JORGE ABARCA-QUINONES ${ }^{3}$ \\ ${ }^{1}$ Institute for Advanced Research, Tarapacá University, Arica 1001236, Chile; \\ ${ }^{2}$ Center for Radiological Research, Columbia University Medical Center, New York, NY 10032, USA; \\ ${ }^{3}$ School of Medicine, Saint-Luc Hospital, IMAG Unit (IREC), University of Louvain, Brussels 1200, Belgium
}

Received March 1, 2016; Accepted April 15, 2016

DOI: $10.3892 / \mathrm{ol} .2016 .4461$

\begin{abstract}
Breast cancer, the most common neoplasm in women of all ages, is the leading cause of cancer-related mortality in women worldwide. Markers to help to predict the risk of progression and ultimately provide non-surgical treatment options would be of great benefit. At present, there are no available molecular markers to predict the risk of carcinoma in situ progression to invasive cancer; therefore, all women diagnosed with this type of malignancy must undergo surgery. Breast cancer is a heterogeneous complex disease, and different patients respond differently to different treatments. In breast cancer, analysis using immunohistochemical markers remains an essential component of routine pathological examinations, and plays an import role in the management of the disease by providing diagnostic and prognostic strategies. The aim of the present study was to identify a marker that can be used as a prognostic tool for breast cancer. For this purpose, we firstly used an established breast cancer model. MCF-10F, a spontaneously immortalized breast epithelial cell line was transformed by exposure to estrogen and radiation. MCF-10F cells were exposed to low doses of high linear energy transfer (LET) $\alpha$ particles $(150 \mathrm{keV} / \mu \mathrm{m})$ of radiation, and subsequently cultured in the presence of $17 \beta$-estradiol. Three cell lines were used: i) MCF-10F cells as a control; ii) Alpha5 cells, a malignant and tumorigenic cell line; and iii) Tumor2 cells derived from Alpha5 cells injected into nude mice. Secondly, we also used normal, benign and malignant breast specimens obtained from biopsies. The results revealed that the MCF-10F cells were negative for c-Ha-Ras protein expression; however, the Alpha5 and Tumor 2 cell lines were positive for c-Ha-Ras protein expression. The malignant breast samples were also strongly positive for c-Ha-Ras expression. The findings of our study indicate that c-Ha-Ras protein expression may be used as a marker to predict the progression of breast cancer; this marker may also ultimately provide non-surgical treatment options for patients who are at a lower risk.
\end{abstract}

Correspondence to: Dr Gloria M. Calaf, Institute for Advanced Research, Tarapacá University, Calle Antofagasta 1520, Arica 1001236, Chile

E-mail: gmc24@columbia.edu

Key words: c-Ha-Ras, breast cancer risk

\section{Introduction}

Breast cancer is a progressive disease and the most common neoplasm affecting women of all ages. Similar to all progressive diseases, early and reliable diagnosis is the key to successful treatment. Benign epithelial breast disease represents a growing percentage of the pathological characteristics of this disease, which include numerous benign entities such cysts, fibrosis, adenosis and duct ectasia, which require neither surgery nor follow-up $(1,2)$. Lesions such as as papillomas, sclerosing adenosis, lobular intraepithelial neoplasia, flat epithelial atypia, radial scars and atypical hyperplasias, which are all considered pre-malignant lesions, are signs of an increased risk of breast cancer (3-7).

It has been demonstrated that human malignant tumors of the breast have an elevated expression of the Harvey Ras oncogene when compared to their respective normal tissue samples (7). In a previous study, the authors performed a comparative analysis of Harvey Ras oncogene expression with conventional clinicopathological parameters of breast cancer (8). In another study, an immunohistochemical analysis of Ras oncogene expression in human breast lesions was performed (9). A high expression of p21 Ras oncogene in breast cancer patients is considered to have important clinical significance (10).

In breast cancer, the increased expression and/or activation of Ras are often associated with tumor aggressiveness $(10,11)$. Available and emerging immunohistochemical and molecular studies have improved the classification of breast cancer, as well as the prognostic and predictive information regarding breast cancer pathology; thus, a new tumor classification has been proposed (12-17). In this new classification, the basal-like and the triple-negative types of breast cancer have a likelihood of distant recurrence and mortality compared with other types, and have a tend to affect younger women. For both subtypes, a need for effective biological markers has been reported in certain studies; thus, Ras expression may prove to be an effective prognotic marker $(13,17)$.

The complexity of breast cancer pathology remains a challenge for the scientific community; therefore, protein biomarkers are needed as indicators of pathological, physiological, or pharmacological processes. Among these, estrogen receptor (ER) and Her2/neu are biomarkers that have been approved for the prognosis, diagnosis and treatment of this disease. Since breast cancer is a heterogeneous disease, some breast cancer cells lose their ability to express ER $\alpha$ among other proteins, resulting in 
a disease which is therapy-resistant. To identify human breast cancer biomarkers between ER $\alpha$-positive and -negative breast cancer, tissues need to be micro-dissected and protein expression can then be identified to be compared with either normal ductal epithelium or ductal epithelium containing ductal carcinoma in situ lesions. Ras and Her2/neu protein expression have been previously considered as biomarkers, since they are highly expressed in human breast cancer (11).

Breast cancer is a heterogeneous complex disease, a spectrum of many subtypes with distinct biological features, and this leads to differences in response patterns to various treatment modalities and clinical outcomes. In this context, the analysis of breast cancer using immunohistochemical markers remains an essential component of routine pathological examinations, and plays an important role in the management of the disease as regards diagnostic, prognostic and therapeutic strategies (18-22). Ras family members (H-Ras, K-Ras, N-Ras and M-Ras) are small GTPases which are activated indirectly by external stimuli. Since the cloning of HRas, the first human oncogene, the Ras/MPK pathway has been a preferential subject of cancer research $(23,24)$, and it is known as an important pathway in the initiation and progression of cancer. Therefore, the aim of the present study was to evaluate Ras expression by immunohistochemical analysis in breast cell lines, as well as in normal, benign and malignant breast sample biopsies, in order to identify a marker that may be used as a prognostic tool for breast cancer patients.

\section{Materials and methods}

Breast cancer cell lines. An in vitro experimental breast cancer model (Alpha model), previously developed by our group by exposing the immortalized human breast epithelial cell line, MCF-10F, to low doses of high linear energy transfer (LET) $\alpha$ particles radiation $(150 \mathrm{keV} / \mu \mathrm{m})$ and subsequent growth in the presence or absence of $17 \beta$-estradiol (25), was used in this study. This model consisted of human breast epithelial cells in different stages of transformation: i) a control cell line, MCF-10F; ii) a malignant and tumorigenic cell line termed Alpha5 (60 cGy plus estrogen/60 cGy plus estrogen); and iii) Tumor 2 cells derived from cells originating from a tumor following the injection of the Alpha5 cell line into nude mice. The cells were grown in DMEM/F-12 (1:1) medium supplemented with antibiotics [100 U/mI penicillin, $100 \mu \mathrm{g} / \mathrm{ml}$ streptomycin, $2.5 \mu \mathrm{g} / \mathrm{ml}$ amphotericin B (all from Life Technologies, Grand Island, NY, USA)], $10 \mu \mathrm{g} / \mathrm{m} \mathrm{5 \%}$ equine serum (Biofluids, Rockville, MD, USA), $0.5 \mu \mathrm{g} / \mathrm{ml}$ hydrocortisone (Sigma-Aldrich, St. Louis, MO, USA) and $0.02 \mu \mathrm{g} / \mathrm{ml}$ epidermal growth factor (Collaborative Research Inc., Bedford, MA, USA).

Protein expression by immunoperoxidase staining. Exponentially growing cell line cells were plated on a glass chamber slide (Nunc Inc., Naperville, IL, USA), at a density of $1 \times 10^{4}$ cells $/ \mathrm{ml}$ of medium and allowed to grow for 2-3 days until $70 \%$ confluent. The cells were fixed with buffered paraformaldehyde at room temperature, incubated with $1 \% \mathrm{H}_{2} \mathrm{O}_{2}$ in methanol to block endogenous peroxidase and again washed twice with buffer solution. Subsequently, the cell cultures were then covered with normal horse serum for $30 \mathrm{~min}$ at room temperature and incubated with either anti-mouse or anti-goat monoclonal or polyclonal antibodies: ER $\alpha$ (mouse, sc-8002), Neu (rabbit, sc-284) and H-Ras (mouse, sc-29) (all from Santa Cruz Biotechnology, Inc., Santa Cruz, CA, USA) at 1:500 dilution overnight at $4^{\circ} \mathrm{C}$ and then incubated for $45 \mathrm{~min}$ with diluted biotinylated secondary antibody solution (Vector Laboratories Inc., Burlingame, CA, USA) and Vectastin Elite ABC reagent (Vector Laboratories Inc.) was used. The experiments were repeated twice in cells with identical passages in vitro.

Breast samples. All samples were obtained from archives entrusted by Professor P. Maldague and Professor A. Trouet from the School of Medicine, Saint-Luc Hospital, IMAG Unit (IREC), University of Louvain, Brussels, Belgium. This study was approved by the Ethics Committee of the University of Louvain and was conducted in accordance with institutional guidelines. The study consisted of the analysis of a total of 40 samples from the archives (all patients had provided with written informed consent prior to obtaining the samples). Normal tissues were obtained from 10 patients admitted for reduction mammoplasty with a family history for cancer; 15 samples had benign breast lesions and 15 specimens had breast cancer. All samples were diagnosed and classified according to the World Health Organization classification (26).

Immunohistochemistry. Processing of the breast samples was performed following a routine pathological examination. All samples investigated were tested for mouse H-Ras monoclonal antibody (Santa Cruz Biotechnology, Inc.). Immunolocalization was performed using a streptavidin-biotin immunoperoxidase method according to the protocol of the laboratory. Briefly, $5 \mu \mathrm{m}$-thick sections obtained after formalin fixation and paraffin-embedding were deparaffinyzed in xylene and rehydrated with Tris-buffered saline (TBS). The sections were then treated with citrate buffer ( $\mathrm{pH}$ 6.0) and hydrogen peroxide was used to quench endogenous peroxidase activity. The slides were then subjected to the primary antibody solution and placed in a moist chamber overnight at $4^{\circ} \mathrm{C}$. After washing in TBS, a biotinylated link antibody was applied, followed by washing and the addition of streptavidin peroxidase. The localization of the antibody was visualized using 3,3'-diaminobenzidine tetrahydrochloride (DAB) and counterstaining with Mayer's hematoxylin (Sigma-Aldrich).

\section{Results}

The established breast cancer model (25) was shown to exhibit several characteristics of breast carcinogenesis. The normal cell line, MCF-10F, did not exhibit any of the features that characterize malignant cells, such as anchorage-independent growth in soft agar, invasion and tumor growth in nude mice. The Alpha5 cell line induced the development of mammary gland tumors in animals after injection and gave rise to the Tumor 2 cell line (25). Fig. 1 shows the results of immunoperoxidase staining obtained to detect ER $\alpha, \mathrm{ErbB} 2$ and c-Ha-Ras protein expression in the MCF-10F, Alpha5 and Tumor2 cell lines. The results indicated a positive expression in the Alpha5 and Tumor 2 cell lines for all 3 markers. However, a negative expression was observed in the MCF-10F cell line. The Tumor2 malignant cells derived from the mice were positive for ER $\alpha$, 


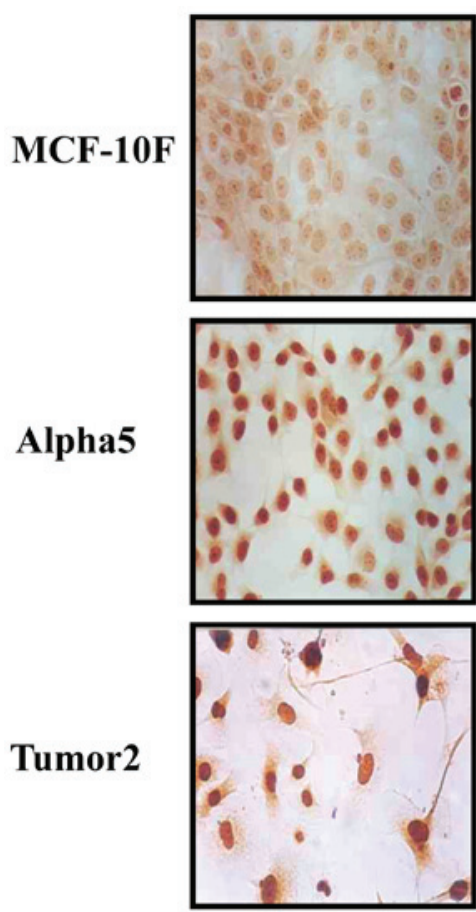

$\mathbf{E R} \alpha$
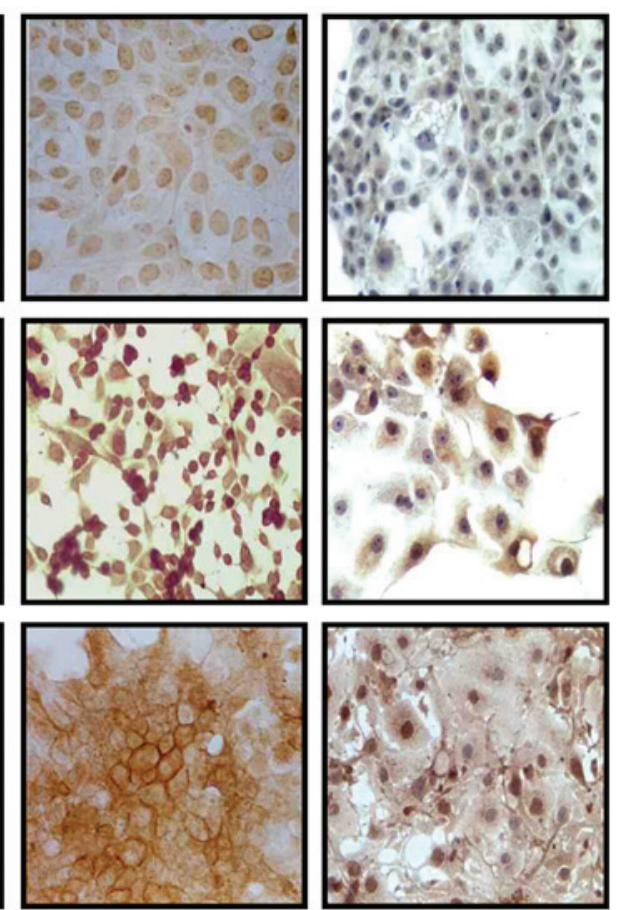

ErbB2

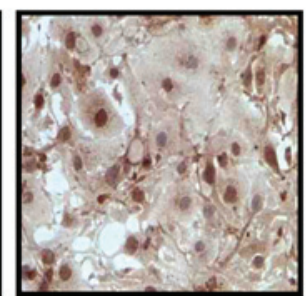

c-Ha-Ras

Figure 1. Representative images of estrogen receptor $\alpha(\mathrm{ER} \alpha)$, Neu and c-H-Ras protein expression determined by peroxidase staining in the MCF-10F, Alpha5 and Tumor2 cell lines. Primary antibodies used were mouse monoclonal antibodies to ER $\alpha$, Neu and H-Ras.

ErbB2 and c-Ha-Ras protein expression, as with the original cell line, Alpha5.

An analysis of breast specimens obtained from the tissue archives of patients with a family history of breast cancer was also carried out. A routine pathological examination revealed marked fibrosis, a variety of cysts with or without apocrine metaplasia, some microcalcifications, some low duct hyperplasias and sporadic epithelial flat lesions in both groups. Our findings support the concept of the heterogeneity of expression in epithelial lesions and the value of immunohistochemical analysis in histopathological diagnosis. Representative images are shown in Figs. 2 and 3. The results indicated that case 1 (without a family history) was a low ductal hyperplasia. Proliferative ducts originating from small structures were observed (see inset). Ducts and ductules were positive for ER $\alpha$ and ErbB2; however, a negative c-Ha-Ras expression was observed in ducts and enlarged ductules. Case 2 (without a family history) exhibited lobules that were negative for ER $\alpha$, ErbB2 and c-Ha-Ras. Case 3 (with a family history) exhibited ducts and ductules positive for ER $\alpha$ and ErbB2; however, the ductules were negative for c-Ha-Ras. Case 4 (with a family history) exhibited lobules negative for $\mathrm{ER} \alpha$, but positive for ErbB2 and c-Ha-Ras. Case 5 (with a family history) also exhibited lobules negative for ER $\alpha$ and $\mathrm{c}-\mathrm{Ha}$-Ras, but positive for ErbB2. Representative images of benign lesions are illustrated in Fig. 2. Insets (when available for each image) show other structures also present in that case, as ducts and ductules.

As shown in Fig. 3, the results indicated that case 6 (with a family history) was a lobular hyperplasia where the lobules were negative for ER $\alpha$, but positive for ErbB2 and c-Ha-Ras. Case 7 (with a family history) also had lobules negative for ER $\alpha$, but positive for ErbB2 and c-Ha-Ras. Case 8 (with a family history) corresponded to ductal carcinoma where the cells were positive for ER $\alpha, E r b B 2$ and c-Ha-Ras. Case 9 (with a family history) exhibited structures positive for ER $\alpha$, ErbB2 and c-HaRas. Case 10 (with a family history) exhibited hyperplastic foci with structures negative for ER $\alpha$, ErbB2 and c-Ha-Ras. Representative images of breast lesions are shown in Fig. 3. The results revealed normal or benign structures, apart from case 3; however, the report stated that those patients had malignant lesions. On the other hand, there was no association with the markers used clinically, as ER and ErbB2 protein expression were positive in benign, as well as in malignant breast lesions with or without hereditary predisposition. The present study revealed that the percentage of cells positive for ER $\alpha$ was low in normal mammary glands and non-proliferative benign breast disease or displasias, but increased in certain malignant lesions.

Representative images of $\mathrm{H}$-Ras protein expression in normal (Fig. 4A) benign (Fig. 4B) and malignant (Fig. 4C) breast lesions are shown. The expression of $\mathrm{H}$-Ras was negative in the normal breast samples, but weakly positive and heterogenous in ductules and ducts. In the benign breast samples, the cyst epithelium was usually positive, with some differential patterns. In most cases, it was distributed in the cytoplasm and occasionally with membrane localization. The apocrine metaplasia foci exhibited a stronger expression, with different patterns between the samples analyzed. A significant increase in immunostaining was observed in the atypical ductal hyperplastic lesions, associated with epithelial ductal proliferation. The cancer specimens revealed a more variable staining pattern. The expression of H-Ras was almost invariably stronger in the cancer specimens than in the benign epithelium from the same patients. Some heterogeneity of expression was observed, with weakly positive or negative cancer cells adjacent to strongly 


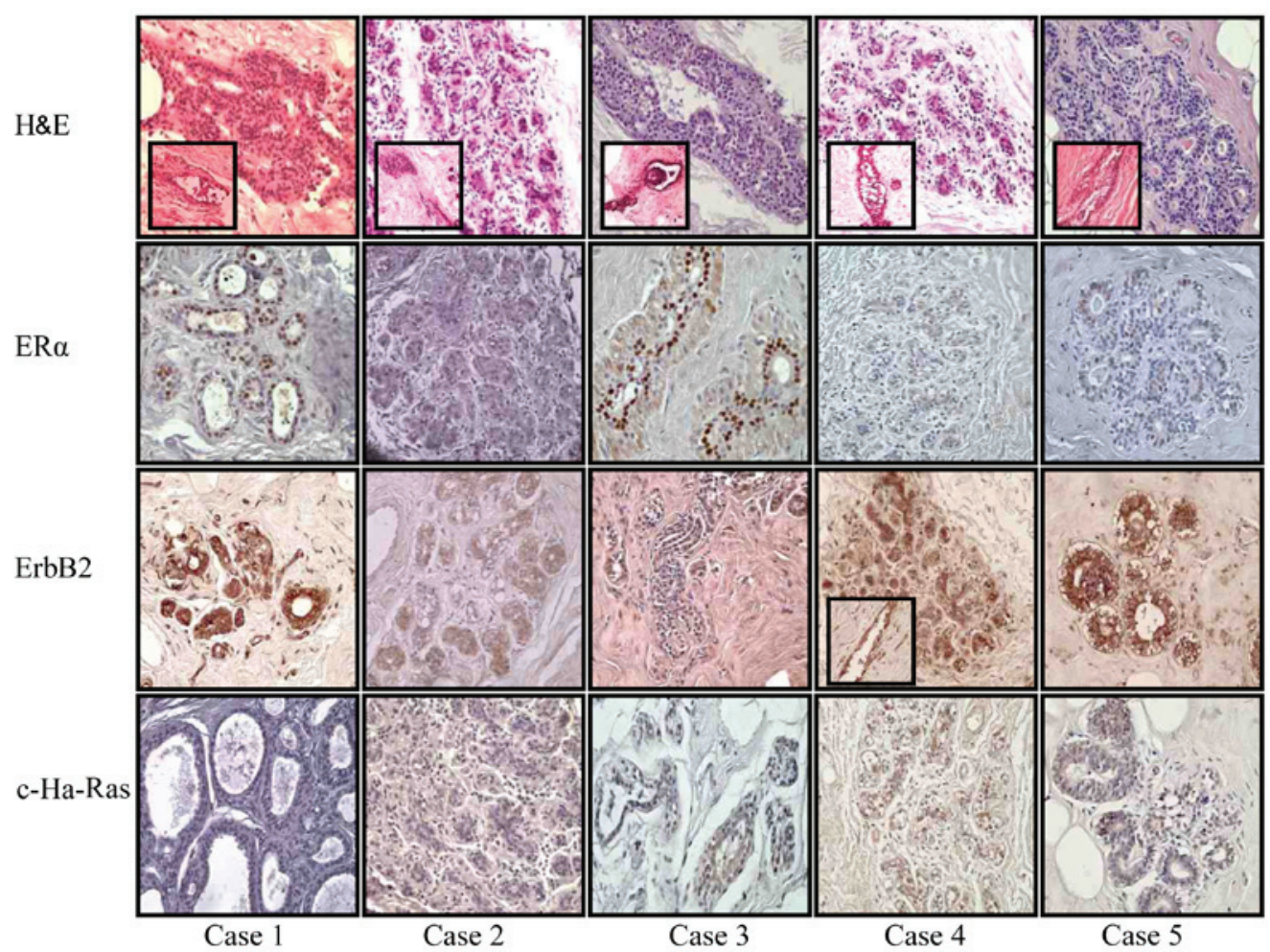

Figure 2. Representative images of estrogen receptor $\alpha(E R \alpha)$, Neu and H-Ras protein expression in breast cancer specimens (cases 1-5) determined by peroxidase staining. Primary antibodies used were mouse monoclonal antibodies to ER $\alpha$, Neu and H-Ras.

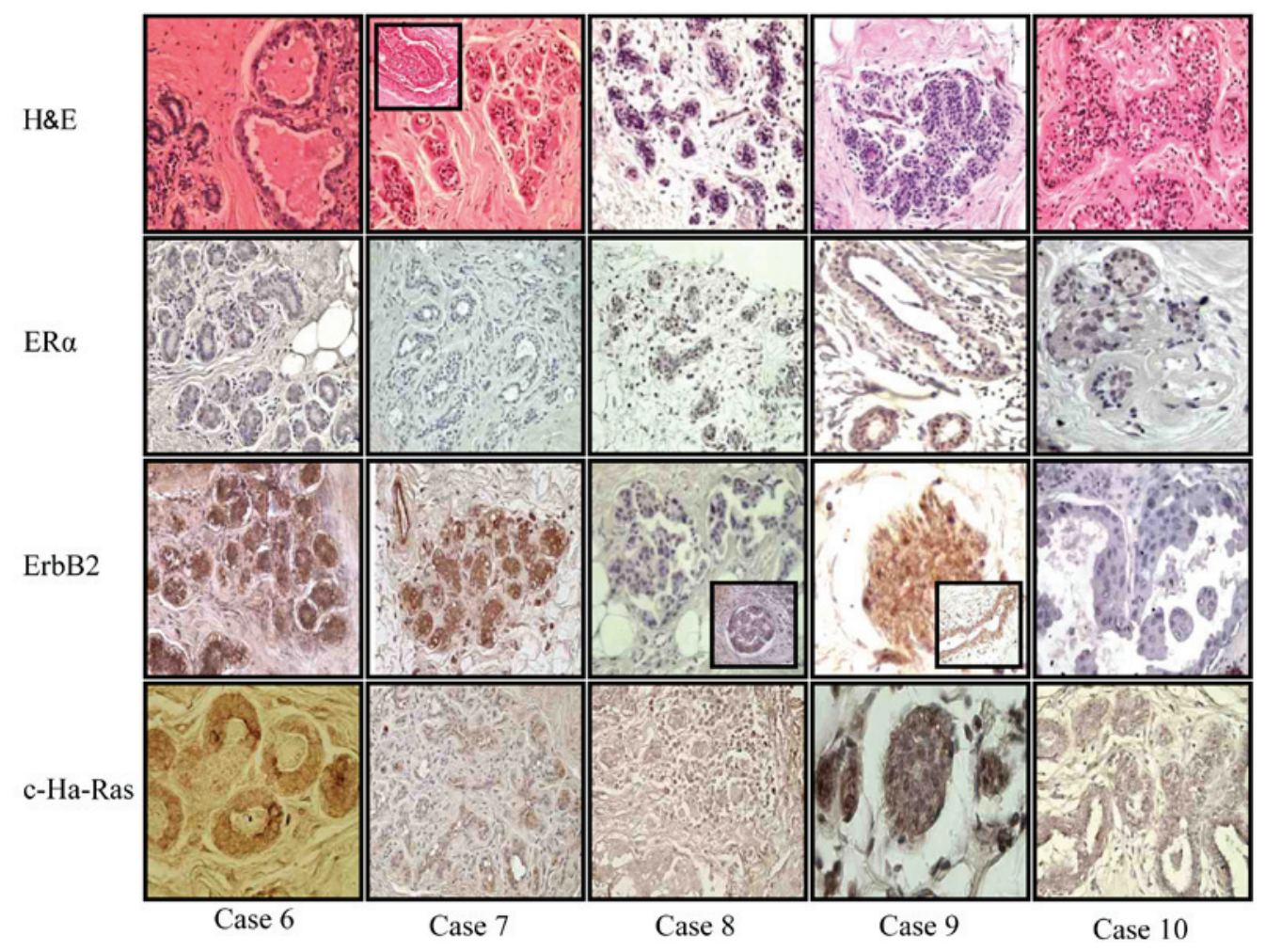

Figure 3. Representative images of estrogen receptor $\alpha(\mathrm{ER} \alpha)$, Neu and H-ras protein expression in breast cancer specimens (cases 6-10) determined by peroxidase staining. The primary antibodies used were mouse monoclonal antibodies to ER $\alpha$, Neu and H-ras.

positive cells. Metastatic cancer cells within the stroma (clusters and isolated cells) exhibited a variable H-Ras expression. Only the tumor budding (clumps) directly associated with carcinoma foci were more homogeneous, with similar patterns of tumor foci. The expression of H-Ras was similar in lobular and ductal carcinomas. 


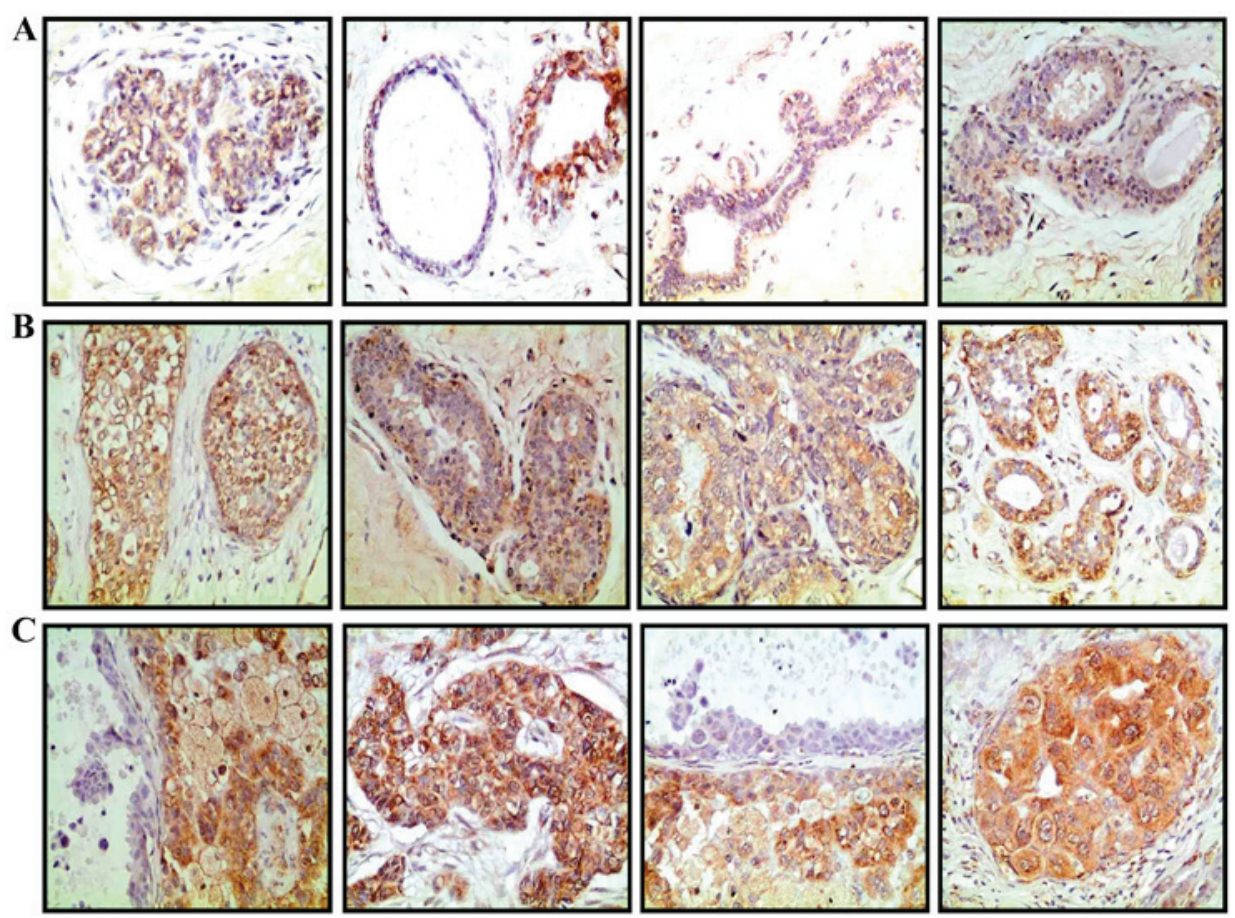

Figure 4. Representative images of c-Ha-Ras protein expression in (A) normal, (B) benign and (C) malignant and breast cancer specimens determined by peroxidase staining. The primary antibody used was mouse monoclonal antibody to H-Ras.

\section{Discussion}

As with all progressive diseases, the early and reliable diagnosis is key to successful treatment. In our analyses, a previously established breast cancer model (25) was used. The normal cell line, MCF-10F, exhibited a negative protein expression of $\mathrm{ER}, \mathrm{ErbB} 2$ and c-Ha-Ras, whereas the malignant Alpha5 and Tumor2 cell line exhibited a positive expression ER, ErbB2 and c-Ha-Ras. This model of progression indicated that malignancy can influence the positivity of these markers, as was also observed in our biopsy specimens. Immunohistochemistry plays is an important diagnostic adjunct to morphological examination in the diagnosis of benign and malignant lesions $(2,7,17,20)$. Markers to help predict the risk of progression, and to ultimately provide non-surgical treatment options to those at lower risk would be of great benefit. At present, there are no applied molecular markers available to predict the risk of carcinoma in situ progression to invasive cancer, and therefore, all women diagnosed with such a malignancy must undergo surgery.

Our results revealed positive c-Ha-Ras protein expression only in the malignant lesions in comparison to the benign lesions. The high c-Ha-Ras expression in intraductal proliferative lesions is very relevant. These epithelial lesions have a risk of progression to invasive breast cancer and related issues, particularly atypical ductal hyperplasia (4). However, the use of c-Ha-Ras expression as a diagnostic and prognostic marker should be selective and should preferably be used in conjunction with other markers. According to published data, the major value of c-Ha-Ras expression is in its clinical correlation with an improved prognosis of relapse $(11,24)$.

In a previous study, the expression of transformation phenotypes was examined in the human breast epithelial cell line,
MCF-10F, with the chemical carcinogens, 7,12-dimethylbenz[a] anthracene, N-methyl-N-nitrosourea, N-methyl-N-nitro$\mathrm{N}$-nitrosoguanidine and benzo[a]pyrene (27). This was done to clarify whether chemically induced neoplastic transformation correlates with alterations in the Ras gene. MCF-10F cells have two c-Ha-Ras alleles, identified by $1.0-$ and $1.2-\mathrm{kb}$ restriction fragments. Treatment with carcinogens resulted in the loss of one of the alleles $(1.0 \mathrm{~kb})$. Polymerase chain reaction-amplified DNA from all carcinogen-treated cells was analyzed for point mutations in c-Ha-Ras at codons 12 and 61 . All of the carcinogens induced a mutation of the remaining allele at the first position of codon $12(\mathrm{GGC} \rightarrow \mathrm{AGC})$. Another frequent mutation occurred at the first position of codon $61(\mathrm{CAG} \rightarrow \mathrm{GAG})$. The changes in c-Ha-Ras were associated with the emergence of colony formation in agar-methocel, but no specific changes in this gene correlated with the emergence of invasiveness or tumorigenesis, indicating that other genes may be involved in the process.

It has been concluded that c-Ha-Ras induces an additive effect on the expression of tumorigenesis in the human breast epithelial cell line, MCF-10F, treated with chemical carcinogens. This has provided a model for analyzing the role of c-Ha-Ras in human breast cancer (28). Data have also highlighted the importance of chromosome 11 in the radiationinduced malignant transformation of human breast epithelial cells and suggest the usefulness of the model in uncovering specific derangements during breast cancer progression (29). Among the loci of chromosome 11, the locus 11p15.5, where the c-Ha-Ras oncogene is located, had an incidence of allelic imbalance between 25 and $40 \%$. Furthermore, direct sequencing analysis of codons 12 and 61 of the c-Ha-Ras oncogene identified various point mutations (29). On the other hand, studies have shown that the c-Ha-Ras oncogene is upregulated by the 
effect of malathion alone and by the combination of estrogen with either malathion or parathion (30). This suggests that pesticides and estrogens affect human breast cells, inducing molecular changes indicative of transformation (30).

In this study, Ras protein expression was examined in samples of normal and hyperplasias, as well as malignant breast cancer lesions with hereditary predisposition in conjunction with other markers regularly used in clinical practice. Since Ras is often mutated in human cancers, much effort has been devoted to devising means of controling the activity of Ras (23). Breast tumors have been shown to have an elevated expression of the Harvey Ras oncogene when compared to their respective normal tissue sections (7). The Harvey Ras oncogene has also been shown to have a significant correlation with the clinicopathological characteristics of breast cancer (8). Immunohistochemical analyses of Ras oncogene expression in human breast lesions have been carried out (9), as well as of the p21 Ras oncogene $(10,21,22)$, with a high expression in breast cancer patients indicating clinical significance. It can thus be concluded that the oncogene, c-Ha-Ras, is a good marker to be used in breast cancer in patients and to predict response to therapy in such patients. The evaluation of Ras expression by immunohistochemistry in cell lines in an experimental breast cancer model of transformed cells by environmental substances and estrogen, as well as in normal, benign and malignant breast biopsies from patients, provided evidence that it can be used as good a prognostic tool for breast cancer patients.

\section{Acknowledgements}

The technical support of Guiliana Rojas, Georgina Vargas and secretarial assistance and suggestions of Leodán A. Crispin and Richard Ponce-Cusi are greatly appreciated. This study was supported by Grant support FONDECYT no. 1120006 (GMC).

\section{References}

1. Azzopardi JG, Ahmed A and Millis RR: Problems in breast pathology. Major Probl Pathol 11: i-xvi, 1-466, 1979.

2. Dupont WD and Page DL: Risk factors for breast cancer in women with proliferative breast disease. N Engl J Med 312: 146-151, 1985.

3. Tsuchiya A, Kanno M, Nomizu T, Hatakeyama Y, Kimijima I and Abe R: Clinical characteristics of breast cancer patients with family history. Fukushima J Med Sci 44: 35-41, 1998.

4. De Mascarel I and MacGrogan G: Management of breast epithelial atypia. Ann Pathol 27: 182-194, 2007 (In French).

5. de Mascarel I, Debled M, Brouste V, Mauriac L, Sierankowski G, Velasco V, Croce S, Chibon F, Boudeau J, Debant A, et al: Comprehensive prognostic analysis in breast cancer integrating clinical, tumoral, micro-environmental and immunohistochemical criteria. Springerplus 4: 528, 2015.

6. Walker RA, Hanby A, Pinder SE, Thomas J and Ellis IO; National Coordinating Committee for Breast Pathology Research Subgroup: Current issues in diagnostic breast pathology. J Clin Pathol 65: 771-785, 2012.

7. Spandidos DA and Agnantis NJ: Human malignant tumours of the breast, as compared to their respective normal tissue, have elevated expression of the Harvey ras oncogene. Anticancer Res 4: 269-272, 1984

8. Agnantis NJ, Parissi P, Anagnostakis D and Spandidos DA: Comparative study of Harvey-ras oncogene expression with conventional clinicopathologic parameters of breast cancer. Oncology 43: 36-39, 1986.

9. Agnantis NJ, Petraki C, Markoulatos P and Spandidos DA: Immunohistochemical study of the ras oncogene expression in human breast lesions. Anticancer Res 6: 1157-1160, 1986.
10. Efremidis AP, Agnantis NJ, Patra F, Papadopoulou C and Spandidos DA: Clinical significance of elevated p21 ras oncogene expression in breast cancer patients. Cancer J 2: 288-291, 1989.

11. Spandidos DA, Yiagnisis M, Papadimitriou K and Field JK: Ras, c-myc, and c-erbB-2 oncoprotein expression in human breast carcinomas. Anticancer Res 9: 1385-1394, 1989.

12. Gruver AM, Portier BP and Tubbs RR: Molecular pathology of breast cancer: The journey from traditional practice toward embracing the complexity of a molecular classification. Arch Pathol Lab Med 135: 544-557, 2011.

13. Park S, Koo JS, Kim MS, Park HS, Lee JS, Lee JS, Kim SI and Park BW: Characteristics and outcomes according to molecular subtypes of breast cancer as classified by a panel of four biomarkers using immunohistochemistry. Breast 21: 50-57, 2012.

14. Perou CM, Sørlie T, Eisen MB, van de Rijn M, Jeffrey SS, Rees CA, Pollack JR, Ross DT, Johnsen H, Akslen LA, et al: Molecular portraits of human breast tumours. Nature 406: 747-752, 2000.

15. Lerwill MF: Current practical applications of diagnostic immunohistochemistry in breast pathology. Am J Surg Pathol 28: 1076-1091, 2004.

16. Abdel-Fatah TM, Powe DG, Hodi Z, Reis-Filho JS, Lee AH and Ellis IO: Morphologic and molecular evolutionary pathways of low nuclear grade invasive breast cancers and their putative precursor lesions: Further evidence to support the concept of low nuclear grade breast neoplasia family. Am J Surg Pathol 32: 513-523, 2008.

17. Moriya T, Kanomata N, Kozuka Y, Fukumoto M, Iwachido N, Hata S, Takahashi Y, Miura H, Ishida K and Watanabe M: Usefulness of immunohistochemistry for differential diagnosis between benign and malignant breast lesions. Breast Cancer 16: 173-178, 2009.

18. Dewar R, Fadare O, Gilmore H and Gown AM: Best practices in diagnostic immunohistochemistry: Myoepithelial markers in breast pathology. Arch Pathol Lab Med 135: 422-429, 2011.

19. Leong AS and Zhuang Z: The changing role of pathology in breast cancer diagnosis and treatment. Pathobiology: Journal of Immunopathology. Mol Cell Biol 78: 99-114, 2011.

20. Omi Y, Yamamoto T, Okamoto T, Obara T and Kobayashi M: A useful immunohistochemical approach to evaluate intraductal proliferative lesions of the breast and to predict their prognosis. Histol Histopathol 26: 79-86, 2011.

21. Candlish W, Kerr IB and Simpson HW: Immunocytochemical demonstration and significance of p21 ras family oncogene product in benign and malignant breast disease. J Pathol 150: 163-167, 1986.

22. Going JJ, Anderson TJ and Wyllie AH: Ras p21 in breast tissue: Associations with pathology and cellular localisation. Br J Cancer 65: 45-50, 1992.

23. Malumbres $\mathbf{M}$ and Barbacid M: RAS oncogenes: The first 30 years. Nat Rev Cancer 3: 459-465, 2003.

24. Giltnane JM and Balko JM: Rationale for targeting the Ras/MAPK pathway in triple-negative breast cancer. Discov Med 17: 275-283, 2014.

25. Calaf GM and Hei TK: Establishment of a radiation- and estrogen-induced breast cancer model. Carcinogenesis 21: 769-776, 2000.

26. Lakhani SR, Ellis IO, Schnitt SJ, Tan PH and van de Vijver MJ (eds): WHO classification of tumors of the breast. International Agency for Research on Cancer, Lyon, 2012.

27. Zhang PL, Calaf G and Russo J: Allele loss and point mutation in codons 12 and 61 of the c-Ha-ras oncogene in carcinogen-transformed human breast epithelial cells. Mol Carcinog 9: 46-56, 1994.

28. Calaf G, Zhang P, Alvarado M, Estrada S and Russo J: C-ha-ras enhances the neoplastic transformation of human breast epithelial-cells treated with chemical carcinogens. Int J Oncol 6: 5-11, 1995.

29. Roy D, Calaf G and Hei TK: Allelic imbalance at 11p15.5-15.4 correlated with c-Ha-ras mutation during radiation-induced neoplastic transformation of human breast epithelial cells. Int J Cancer 103: 730-737, 2003.

30. Calaf GM and Roy D: Cancer genes induced by malathion and parathion in the presence of estrogen in breast cells. Int J Mol Med 21: 261-268, 2008. 